\title{
Developments for lighting applications by using prismatic film
}

Berta García-Fernández, Antonio Álvarez FernándezBalbuena , Daniel Vázquez-Moliní

Berta García-Fernández, Antonio Álvarez Fernández-Balbuena , Daniel Vázquez-Moliní, "Developments for lighting applications by using prismatic film," Proc. SPIE 11120, Nonimaging Optics: Efficient Design for Illumination and Solar Concentration XVI, 111200R (9 September 2019); doi: $10.1117 / 12.2529254$

SPIE Event: SPIE Optical Engineering + Applications, 2019, San Diego, California, United States 


\title{
Developments for lighting applications by using prismatic film
}

\author{
Berta García-Fernández a,b, Antonio Álvarez Fernández-Balbuena a , Daniel Vázquez-Moliní a \\ ${ }^{a}$ Departamento de Óptica, Universidad Complutense de Madrid, Fac de Óptica y Optometría. \\ Arcos de Jalón, 118, 28037 Madrid (SPAIN) \\ ${ }^{\mathrm{b}}$ Departamento de Ingenieria y Gestion Forestal y Ambiental, Universidad Politécnica de Madrid, \\ ETSI de Montes. Ciudad Universitaria s/n 28040 Madrid (SPAIN)
}

\begin{abstract}
Prismatic film offer advantages such as the possibility of transmit light effectively in lighting applications with the benefit of reducing energy consumption. This paper presents several developments based on their characteristics which allow direct light suitably in several fields as architecture, road and automotive lighting and signaling. A mathematical model is presented to provide a quantitative estimation of the influence of numerous factors that affect the transport of light in order to easily predict light extraction while avoiding heavy software calculations. The deformation produced in a prismatic film induced by changes in the curvature of the cylindrical guide is characterized by image analysis. This method shows the relationship between the curvature of the film, the deformation and the flux of transmitted light.
\end{abstract}

Keywords: Prismatic film, light guide, defects, transmittance model.

\section{INTRODUCTION}

Controlling lighting is an essential condition for the improvement of human life's quality, the reduction of the energetic consumption and the cutback of the overall costs. Although new optical fibers of plastic materials, high core diameter transmit light with little attenuation in the visible spectrum there are limited ways to extract the light efficient and controlled during the fiber path [1,2]. Some recent developments within the automobile industry comprising optical guides in which the light beam is propagated [3], including sometimes an output face and one reflection surface comprising different configurations of prisms [4]. Hollow light guides are among the most used guidance systems with minimum loss and can function as a high efficiency luminaire. Within the classification of hollow guides, prismatic guides offer an innovative alternative with minimum loss and excellent quality. Thanks to the microprismatic geometry of the sheet and their high degree of transparency, a large part of the light is directed under the optical principle of Total Internal Reflection. PLGs has been developed by modifying the system geometry according to the objectives [5, 6]. Furthermore, prism rods comprising a reflecting area located and holding a plurality of light-deflecting prisms have been designed with a more homogeneous appearance of lighting surfaces [7]. Therefore, hollow prismatic light guides are optical elements providing a wide range of design alternatives. PLGs are giving better light quality than other guidance alternatives because it changes minimally the color characteristics of the output light at the end of the guide [8].

The transmittance or efficiency of the prismatic guide depends mainly on the size of the guide and the angle of incidence of the incident light. Defects in manufacturing and materials lead to irregularities on the surface modifying the behavior of incident rays, producing consequently a progressive reduction of the luminous flux that is transmitted in prismatic films through TIR $[9,10,11,12]$. Therefore, surface defects are an important factor to take into account in furtherance of their optical development.

In addition to areas such as lighting in architecture, the automotive illumination and display technology has undergone considerable technological advances in recent decades such as the use of LEDs which represents an industry breakthrough in lighting technology and is rapidly becoming the most important innovative technologies around the world in the lighting community $[13,14,15]$.

Nonimaging Optics: Efficient Design for Illumination and Solar Concentration XVI, edited by Roland Winston, Eli Yablonovitch, Proc. of SPIE Vol. 11120, 111200R · (c) 2019 SPIE CCC code: $0277-786 \mathrm{X} / 19 / \$ 21 \cdot$ doi: $10.1117 / 12.2529254$ 
This paper show several developments related to the performance of prismatic sheets. Firstly it is shown a mathematical model based on experimental and theoretical measures to determine the efficiency in the PLGs [11]. Later, it is provides a method of characterizing the deformation produced in a prismatic film induced by changes in the curvature. This method is based on recognition procedures and image analysis. Experimental quantifications evince light losses due those changes [12].

\section{ESTIMATION OF TRANSMISSION EFFICIENCY BY A MATHEMATICAL MODEL}

A mathematical model has been developed to estimate light losses in the prismatic guides by the influence of several parameters: the defects in prismatic vertices, the coefficient of absorptance of the material and the defects of the film. The losses are analyzed through efficiency $T g$, to evaluate the system's capacity in light guidance. The efficiency is defined as:

$$
\operatorname{Tg}=\frac{\theta t}{\theta}
$$

Where $\theta i$ is the total flux that enters into the guide and $\theta t$ is the flux intercepted at different positions of the guide. The variation of a magnitude decreases proportionally according to a negative exponential model. The proposed expression containing several independent terms is:

$$
\operatorname{Tg}(r, \mu, \rho)=e^{-\rho(k 1 r+k 2 \mu+k 3),}
$$

where $\mathrm{r}$ is the radius of the vertices of the prisms, $\mu$ is the absorptance coefficient of the prismatic film and $\rho$ is the aspect ratio of the light guide ( $\rho=$ Length/Diameter). The radius $r$ is considered the main source of losses due to the greater concentration of superficial defects located at the vertices of the prisms of the prismatic film. Absorptance $\mu$ is the second terminus of the equation, its contribution is important to improve the fit. The third variable includes several factors containing residual losses related to defects of the prismatic film: stretch marks, inhomogeneities of the material and other. $k 1, k 2 \mathrm{y} k 3$ are the parameters of adjustment of each term which are obtained by means of theoretical and experimental calculations.

The theoretical results $(k 1$ and $k 2)$ are based on measurements with realistic simulations carried out by the use of the raytracing software called Tracepro 7.4. The third variable $k 3$ is an adjustment value obtained from data evaluated experimentally. The values obtained are: $k 1=2.6103 \mathrm{~m}^{-1}, k 2=5.8103 \mathrm{~m}$ y $k 3=1.3$ $10^{-3}$ (Fig. 1 (a).
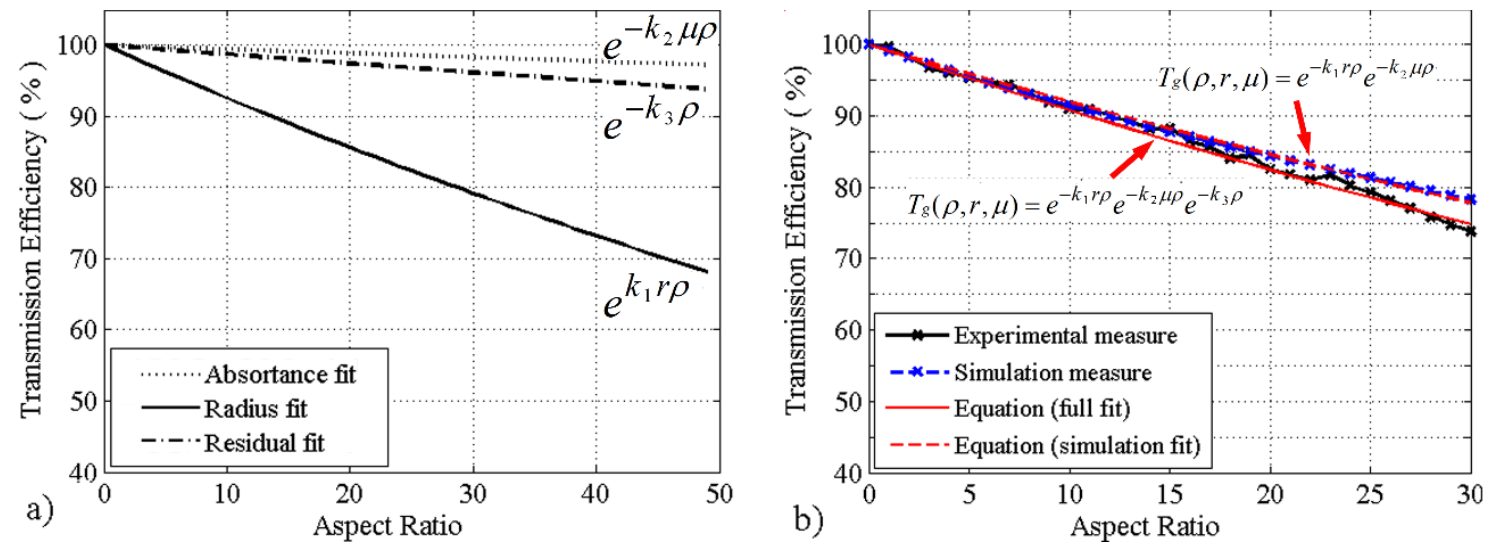

Figure 1 (a) shows the independent contribution of each of the terms of equation 2: the radius in vertices of $3 \mu \mathrm{m}$ (solid line), the absorptance (dotted line) and the residual losses (dotted line with hyphen). (b) Results of the mathematical model of efficiency adjustment, simulations and experimental measurements 
The results in efficiency of the semi-empirical model have been compared with the data of simulations and experimental measurements (Fig. 1 (b). The results of the experimental measurements (solid black line) are superimposed on the model fit of Eq. 2, which is shown by a red continuous line in Figure 1(b) and takes into account all loss factors, that is, $k 1, k 2$ and $k 3$.

\section{PERFORMANCE OF PRISMATIC FILM INDUCED BY CHANGES IN CURVATURE}

\subsection{Deformation of the prismatic angle}

Prismatic sheets must be bent to fit the shape of some surfaces, so changes induced by curving the prismatic film give rise to deformation shifts. An experimental characterization method is provided in order to obtain a detailed analysis of the rate of angular deformation of the prismatic structure due to changes in the geometry for bending the film. Through image processing algorithms, changes in prism apex angle due to the curvature are experimentally evaluated in a transverse section of prismatic film. Those analysis were carried out in seven curvatures, from plane to radius $66.67 \mathrm{~m}^{-1}$.

Firstly, a threshold determined from the grey-level histogram is applied to the input image in order to make it binary. Secondly, the profile edge is obtained using Canny operator [16], this operator is an optimal edge objects and hard edges. Finally, we used the Hough Transform (HT) [17] to detect the parameters that control the accuracy of the right angle at the vertex of the prism.

Figure 2 shows total prismatic angle obtained by HT related with curvature (global changes). The pressure applied to the prismatic geometry at curvatures higher than $20 \mathrm{~m}^{-1}$ increases the deformation in the prism geometry causing the gradient change. The analysis of the mean value of the peaks in the Hough matrix represented as the angular deviation rate is shown in figure $2(\mathrm{~b})$.
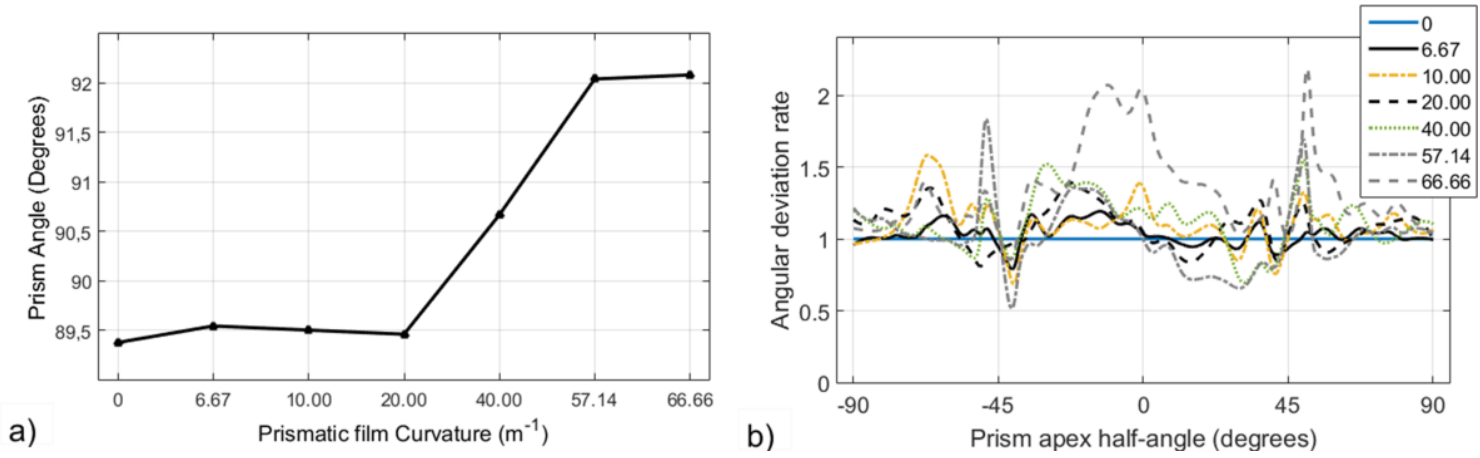

Figure 2. Angle $\theta$ obtained by HT related with CPLG curvature. The graph legend shows curvatures evaluated in m-1. Global changes in prismatic angle related with Hough peaks are shown in (a) and local changes in prism half-angle in (b).

\subsection{Light transmitance in curved Prismatic Film}

Light losses of prismatic film used in PLG, with a range of curvatures from plane to $57.14 \mathrm{~m}^{-1}$ were experimentally measured using physical proto-types on a real size scale to investigate the influence of curvature of prismatic film in flux transmittance.

Light supplied by the He-Ne laser (JDS Uniphase 1508 Helium Neon Gas Laser) is injected into the plane surface of the prismatic film for several incidence angles. The fraction of the total flux loss induced by curvature as a function of the angle of incidence is recorded by a photometer (Gamma Scientific's flexOptometer) The light flux lost rate obtained $\phi k$ (Fig. 3) was estimated by dividing the transmission measurements through the film obtained for each particular curvature $\phi k i$ by the transmission obtained with the corresponding incident flux $\phi t(\phi k=\phi k i / \phi t)$. 


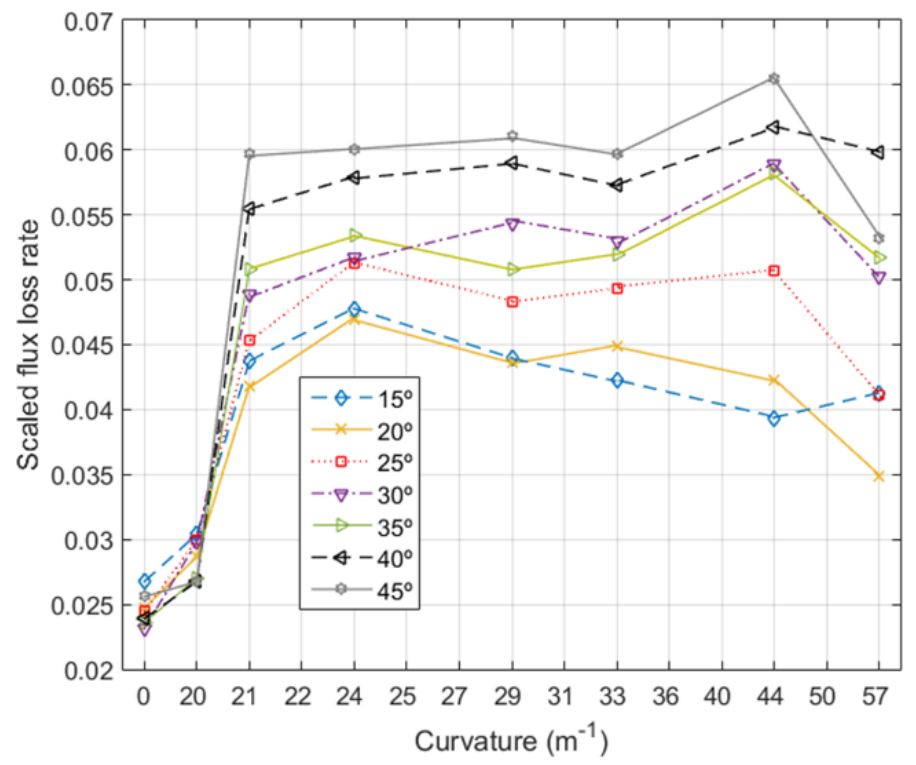

Figure 3. Rate of flux lost scaled from 0 to 1.0 as a function of the curvature of a PLG for several incidence angles. The graph legend shows incidence angles with regard to the prismatic film flat surface.

The light flux rate escaped through the film is minimum in plane structure. After curvature deformation, there is a high increase in flux lost, resulting in a mean loss from 1.5 to $3.2 \%$ in flux loss of PLG with curvatures higher to 20 . Losses increase with the increasing incidence angles.

\section{CONCLUSIONS}

It is important to analyze the failures that limit prismatic film as an optical guidance system, such as, for example, vertices that do not meet the strict angular relationship, surfaces that are not optically smooth and inhomogeneities in the materials used, and alter transmission of light.

It is shown the influence that diverse parameters have on the operation of prismatic sheets as an element of guided light and how they affect efficiency through a mathematical model.

The relation between curvature and lighting efficiency of prismatic sheets is shown. Results demonstrate good agreement between prism deformations and light flux propagation rate obtained by experimental measurements depending on the curvature.

\section{REFERENCES}

[1] Hecht, J.; Long, L. [Understanding fiber optics]. Prentice Hall, 1993.

[2] Remillard, J.T., Everson, M. P. and Weber, W. H. "Loss mechanisms in optical light pipes," Appl. Opt. 31, 7232-7241 (1992).

[3] Teijido, J. M., Herzig, H. P., Daendliker, R., Grupp, J., \& Poli, J. C., "Design of a nonconventional illumination system using a scattering light pipe," In Design and Engineering of Optical Systems (Vol. 2774, pp. 747-757). International Society for Optics and Photonics, (1996, August). 
[4] Thomas L. R. Davenport, "Optimization of prismatic light extraction elements in automotive signal or ambience lighting," Proc. SPIE 10693, Illumination Optics V, 106930T (28 May 2018).

[5] 2301 OLF Data sheet, Available at: http://multimedia.3m.com/mws/media/11326830/3mtm-opticallighting-film-2405.pdf

[6] Alvarez Fernandez-Balbuena, A., Vazquez-Molinì, D., García-Fernandez, B., García-Rodríguez, L., \& Galán-Cañestro, T. "Daylight illumination system by vertical Transparent Prismatic Lightguide for an office building," (2010).

[7] Daniel Vazquez-Molini, Antonio Alvarez Fernandez-Balbuena, Berta Garcia-Fernandez, Eusebio Bernabeu, "High efficiency $90^{\circ}$ elbow for light guides," Proc. SPIE 7059, Nonimaging Optics and Efficient Illumination Systems V, 70590S (11 September 2008).

[8] García-Fernández, B., Vázquez-Molini, D. and Álvarez Fernández-Balbuena, A. "Lighting quality for aluminum and prismatic light guides," Illumination Optics II. Vol. 8170. International Society for Optics and Photonics, (2011).

[9] Min-Wen W. and Chih-Chin T., "Analysis and fabrication of a prism film with roll-to-roll fabrication process," Opt. Express 17, 4718-4725 (2009)

[10] B. Garcia-Fernandez, D. Vazquez-Molini, Alvarez Fernandez-Balbuena, A. and Bernabeu. E. "Light output losses of prism light guides," In SPIE Optical Systems Design, pages 85502Z-85502Z-7. International Society for Optics and Photonics, (2012).

[11] García-Fernández, B., Vázquez, D., Álvarez Fernandez-Balbuena, A., García-Botella, A., MartínezAntón, J. C., "Light losses in hollow, prismatic light guides related to prism defects: a transmittance model," Chinese Optics Letters, 13(9), 092201 (2015).

[12] García-Fernández, B., Fernández-Balbuena, A. Á., \& Vázquez-Moliní, D. "Analytical method to measure bending deformations in prismatic optical films," Chinese Optics Letters, 14(4), 042201, (2016).

[13] Xie, B., Hu, R., Chen, Q., Yu, X., Wu, D., Wang, K., \& Luo, X. "Design of a brightness-enhancementfilm-adaptive freeform lens to enhance overall performance in direct-lit light-emitting diode backlighting," Applied optics, 54(17), 5542-5548, (2015).

[14] Wang, Yi-Jun, et al. "High directional backlight using an integrated light guide plate," Optics Express 23.2 1567-1575, (2015).

[15] Junwon Lee, John E. Greivenkamp, "Modeling of automotive interior illumination systems," Opt. Eng. 43(7) (1 July 2004)

[16] J. Canny, "A computational approach to edge detection," in IEEE Trans. Pattern Analysis and Machine Intelligence 8(6):679-698, (IEEE, 1986).

[17] P.V.C. Hough, "Methods and means for recognizing complex patterns," U.S. Patent 3,069,654 (Dec. 18, 1962). 